\title{
POSITION ANGLES AND ALIGNMENTS OF CLUSTERS OF GALAXIES
}

\author{
Manolis PLIONIS \\ SISSA - International School for Advanced Studies, \\ Via Beirut 2-4, 34013 Trieste, Italy and \\ ICTP - International Centre for Theoretical Physics
}

\begin{abstract}
The position angles of a large number of Abell and Shectman clusters, identified in the Lick map as surface galaxy-density enhancements, are estimated. In total I determine the major axis orientation of 637 clusters out of which 448 are Shectman clusters (202 of which are also Abell clusters) and 189 are Abell clusters not originally detected by Shectman due to his adopted density threshold. Using published redshifts for 277 of these clusters I have detected strong nearest neighbour alignments over scales up to $\sim 15 h^{-1} \mathrm{Mpc}$ at a $\gtrsim 2.5-3 \sigma$ significance level, while quite weak alignments are detected even up to $\sim 60 h^{-1}$ Mpc. A more significant alignment signal $(\sim 4 \sigma)$ is detected among all neighbours residing in superclusters and having separations $\lesssim 10 h^{-1} \mathrm{Mpc}$. Again, weaker but significant alignments are found when larger separations are considered. Since my cluster sample is neither volume limited nor redshift complete, a fact that would tend to wash-out any real alignment signal, the alignments detected should reflect a real and possibly a stronger underline effect.
\end{abstract}

Subject headings: Cosmology - galaxies: clustering - galaxies: formation

Submitted to ApJ Supplements (December 1993) 


\section{Introduction}

The issue of the elongation of clusters of galaxies (cf. Carter \& Metcalfe 1980, Binggeli 1982, Plionis, Barrow \& Frenk 1991) and their tendency to be aligned with their nearest neighbour and in many cases with the position angle of their first ranked galaxy is well studied and well documented, although conflicting results appear occasionally in the literature.

The alignment effect was first noted by Binggeli (1982) who claimed that clusters tend to be aligned with their neighbours over scales of 10-15 $h^{-1} \mathrm{Mpc}$. Since then, a number of authors have supported the reality of this effect (Rhee and Katgert 1987, Flin 1987, West 1989a,b, Lambas et.al. 1990, Rhee, van Haarlem \& Katgert 1992), although doubts have been put forward about the significance and the strength of the effect (Struble \& Peebles 1985; McMillan, Kowalski \& Ulmer 1989; Fong, Stevenson \& Shanks 1990). Note, however, that Fong et.al. searched for alignments between neighbouring clusters found in 2-dimensions. Any real signal could be diluted by projection and therefore one should be cautious on how to interpret these results. In fact, as it will be shown in what follows, a significant alignment signal between neighbours in 3 dimensions becomes insignificant, although still present, when the cluster pairs are chosen in angular space.

Further support for the reality of the alignment effect comes from the work of Argyres et al (1986) and Lambas, Groth \& Peebles (1988) who found that the Lick galaxy counts around Abell clusters tend to be aligned with the cluster major axis out to $\sim 15 h^{-1} \mathrm{Mpc}$, especially for clusters in high density regions.

It was thought initially that the observed alignment effects would provide a very effective test to discriminate among different models of cosmic structure formation. In the pancake scenario (Zeldovich 1970, Doroshkevich, Shandarin \& Saar 1978), like the Hot Dark Matter model, where clusters and galaxies form by fragmentation in already flattened sheet- and filament-like superclusters, one expects the clusters to be elongated and aligned. In accordance with this Dekel, West \& Aarseth (1984) and West, Dekel \& Oemler (1989) found that cluster alignments occur only when the initial fluctuation spectrum has a large coherence length as that expected in the HDM model. They where unable to reproduce the observed alignments in hierarchical clustering models, like the Cold Dark Matter (CDM) model, where the cosmic structures form by gravitational clustering from small to large scales (cf. Peebles 1982, Blumenthal et.al. 1984, Davis et.al. 1985, Frenk et.al. 1985, 1988). However, both the asphericity of clusters and the alignment effect could be produced in hierarchical models by a different mechanism. Tidal effects could influence the shapes of protoclusters and induce alignments. Indeed, Binney \& Silk (1979) found that tidal effects between protostructures can induce flattening and prolate shapes for clusters with a mean ellipticity before virialization is $\langle\varepsilon\rangle \approx 0.5$, which is in good agreement with observations (Plionis, Barrow \& Frenk 1991). However this issue is a controversial one since conflicting results have been presented in the literature. For example Barnes \& Efstathiou (1987) using N-body simulations find that tidal interactions cannot induce alignments between neighboring protoclusters while Salvador-Sole \& Solanes (1993) find, using analytical methods, that tides can induce the observed alignments. 
Aside of these conflicting results and contrary to the numerical work of Dekel et.al. (1984) and West et.al. (1989), Bond (1987) has shown that within the framework of Gaussian statistics and if the clusters form at the peaks of the field then an alignment up to $\sim 20 \mathrm{~h}^{-1} \mathrm{Mpc}$ should be expected even in the CDM model. Soon after, analysis of new high-resolution CDM simulations showed that the alignment effect is present and even stronger than what was anticipated by the analytical work (West, Villumsen \& Dekel 1991 and references therein). In view of these results, it would seem unavoidable to conclude that that cluster-cluster alignments is a generic feature of the cluster formation process which is independent of the specific model and form of the fluctuation spectrum. Therefore, cluster alignments cannot be used as an effective discriminant between models of structure formation. However, studying alignments and similar features of the distribution of matter on large scales, could provide interesting clues about the details of the cluster formation process and therefore it is essential:

1. To unambiguously determine whether alignments do occur in the real universe.

2. To find the amplitude of the effect and the scale over which it takes place.

3. To identify details of the alignment effect, for example whether galaxies within a cluster exhibit any alignment (cf. Struble 1990, van Kampen \& Rhee 1990, Trevese, Cirimele \& Flin 1992, Rhee, van Haarlem \& Katgert 1992), which could then provide clues about the internal dynamics of the cluster (cf. Rhee \& Roos 1990).

In this paper I present new cluster position angle determinations for a sample of 637 clusters of galaxies and I study the cluster alignment properties for a subsample of these clusters $(N=277)$ for which redshift information is available.

\section{Cluster Position Angles}

The cluster-finding algorithm, used to identify clusters from the Lick galaxy catalogue, and the resulting cluster catalogues were presented in Plionis, Barrow \& Frenk (1991) [hereafter PBF]. The algorithm is based in identifying surface galaxy-density peaks above a given threshold and connecting in a unique cluster all neighbouring cells that also fulfill the overdensity criteria. A thorough statistical analysis of the distribution of these clusters was presented in Plionis \& Borgani (1992), Borgani, Jing \& Plionis (1992) and Borgani, Plionis \& Valdarnini (1993). I therefore refer the interested reader to those articles as well as to Shane \& Wirtanen (1967) and Seldner et.al. (1977) for issues related to the Lick galaxy catalogue and to Plionis (1988) for details of this particular use of the catalogue. I will just remind the reader that the Lick catalogue contains $\sim 810000$ unique galaxies with $m_{b} \lesssim 18.8$ which are binned in $10 \times 10 \operatorname{arcmin}^{2}$ cells. The catalogue covers $70 \%$ of the sky and its characteristic depth is $210 h^{-1} \mathrm{Mpc}$ (Groth \& Peebles 1977).

I determine the orientation of a cluster by estimating its position angle, $\theta$, measured relative to North in the anticlockwise direction. The principal axes of each cluster and their orientation are calculated by diagonalizing the $2 \times 2$ inertia tensor $\mathbf{I}$ : 


$$
I_{k l}=\sum_{i=1}^{n}\left(r_{k} r_{l}\right)_{i} m_{i}
$$

where $k, l=1,2, r_{1_{i}}=x_{i}, r_{2_{i}}=y_{i}, m_{i}$ is the galaxy number-count of the $i^{t h}$ cell, $n$ is the number of $10^{\prime} \times 10^{\prime}$ cells for each cluster, and the origin of the coordinate axis is the centre of mass of the cluster. This method for determining cluster position angles is quite different from those used in most other studies (cf. Binggeli 1982) which measure the galaxy distribution within a fixed circular aperture around each cluster. This method has the disadvantage that it does not map the same area around each cluster but it has the advantage that it avoids any biases resulting from the use of a circular aperture (for a detailed discussion of this see Carter \& Metcalfe 1980 and Binggeli 1982.)

My primary sample consists of all C36 clusters, ie., those with $\sigma /\langle\sigma\rangle=3.6$ (see PBF), which sample a large enough area to make the determination of their position angle possible, ie., cover at least five $10 \times 10 \operatorname{arcmin}^{2}$ cells. Note that the overdensity threshold used is the same as that used by Shectman (1985) and therefore these clusters correspond to Shectman clusters. In order to test the robustness of the position angle determination the clusters are traced to lower overdensity levels $(\sigma /\langle\sigma\rangle=3,2.5$ and 1.8) and at each level their position angles are determined. Since, however, at lower overdensities there is a higher probability of the clusters being affected by projection effects that could distort their position angles and since projection effects should cause the apparent position of the cluster centre to vary from level to level, PBF attempted to minimize this problem adopting the following procedure. At each overdensity level the cluster centre of mass is estimated and only those levels are considered for which the cluster centre of mass, calculated at that overdensity, has shifted by $<10$ arcmin from its original C36 position (ie., the cluster centre of mass should not move out of its original $10^{\prime}$ cell). $\mathrm{I}$ also estimate the position angles of all Abell clusters, found at lower overdensity levels and which cover enough area to make the position angle determination possible. Note that in order to eliminate the gross effects of Galactic extinction the samples are limited to $|b| \geq 40^{\circ}$.

In table 1 I present the C36 cluster position angles with their corresponding Shectman (1985) and Abell (1958) number. Where it was possible I also present the mean and standard deviation of their values from measurements at three, at least, overdensity levels. In such cases the median value is also listed. In table $1 \mathrm{~b} \mathrm{I}$ present the position angles of Abell clusters identified at $\sigma /\langle\sigma\rangle=2.5$ and determined at the same level, while in table 1c the listed position angles are of clusters identified at a level $\sigma /\langle\sigma\rangle=1$.8. Finally at table $1 \mathrm{~d}$ I present position angles of clusters identified at the $\sigma /\langle\sigma\rangle=2.5$ but estimated at $\sigma /\langle\sigma\rangle=1.8$

\section{Tests for systematic effects}

PBF gave a thorough discussion of the most significant systematic biases that could affect the cluster shape parameters and found that the position angles remain mostly unaffected by the effect of the grid while when tracing the clusters to lower overdensity levels they found small variations of their position angle. However, when the cluster sampling area is small (ie, when few $10^{\prime} \times 10^{\prime}$ cells define 
the cluster), then due to the limiting geometry of the possible cell configuration, the position angles of such clusters tend to have a preferred range of values. Figure 1 shows the position angles as a function of the number of cells, defining the cluster sampling area, for clusters with only one determination of their position angle. Especially for $N_{\text {cells }} \leq 7$ the effect is evident. However, the effect is suppressed when more than one determinations of the position angle of a cluster is possible and in these cases the final value of $\theta$ is the average over all the determinations. This can be seen in figure 2 where we plot the position angles of clusters estimated in more than one overdensity level; panel (a) shows the values of $\langle\theta\rangle$ as a function of the minimum number of cells used while panel (b) the values of $\theta$ as a function of the maximum number of cells used for the $\langle\theta\rangle$ determination. I conclude that clusters with one position angle determination and $N_{\text {cells }} \leq 7$, have an uncertainty, due to the grid, of $\delta \theta \sim 30^{\circ}-35^{\circ}$ and therefore the interested reader should be cautious of this effect and should consider their position angles rather as indicative.

To test whether this effect is significant and whether it could create a systematic orientation bias I calculate, following Struble \& Peebles (1985), the following functions:

$$
\begin{gathered}
C_{n}=\sqrt{\frac{2}{N}} \sum_{i=1}^{N} \cos 2 n \theta_{i} \\
S_{n}=\sqrt{\frac{2}{N}} \sum_{i=1}^{N} \sin 2 n \theta_{i}
\end{gathered}
$$

where $n \geq 1$ and $N$ is the total number of clusters in my sample. If the position angles are independently drawn from a uniform distribution between $0^{\circ}$ and $180^{\circ}$ then the $C_{n}$ and $S_{n}$ have zero mean and unit standard deviation. A systematic bias in the cluster orientations will manifest itself as a large value of $\left|C_{n}\right|$ and/or $\left|S_{n}\right|$. For all the 637 cluster position angles I obtain $\left|C_{1}\right|,\left|S_{1}\right| \lesssim 1.6,\left|C_{2}\right|$, $\left|S_{2}\right| \lesssim 1.2$ and $\left|C_{3}\right|,\left|S_{3}\right| \lesssim 1$ indicating that the distribution of position angles has no significant deviation from uniformity. However, when I use clusters with only one position angle determination and with $N_{\text {cells }}=5$, I get $\left|S_{2}\right|,\left|C_{2}\right| \sim 2$ which reflects the grid effect mentioned before.

PBF found that when they compared the position angles of clusters in common with other studies, the typical uncertainty between the different measurements is $\delta \theta \sim 30^{\circ}-35^{\circ}$, in agreement with West (1989). For example the position angle deviation for the clusters in common with Struble \& Peebles (77 clusters) is $\delta \theta \sim 0^{\circ} \pm 35.5^{\circ}$ while when I compare with 61 common clusters of Rhee et.al. (1992) I find $\delta \theta \sim-1.4^{\circ} \pm 40.5^{\circ}$ for those determined by the Fourier method while I find slightly worse results for their other methods. Interestingly, I find the best agreement when I compare my cluster position angles with their $1^{s t}$ rank galaxy position angles $\left(\delta \theta \sim 0.5^{\circ} \pm 39^{\circ}\right)$.

Note, however, that PBF found that if the comparison is restricted only to those common clusters for which their estimate of the position angle is based on an area similar in size to the circular aperture used in other studies then the position angle deviation is reduced by $\sim 50 \%$. This shows that the different position angle estimation methods lead to similar values when applied to similar regions around the clusters. 


\section{Alignments of Clusters}

In order to search for alignments in my sample I collected all the published cluster redshifts to end up with 277 clusters in the North and South galactic hemisphere. For this sample I find $\left|C_{1}\right|,\left|S_{1}\right| \leq 1.2$, $\left|C_{2}\right|,\left|S_{2}\right| \leq 0.2$ and $\left|C_{3}\right|,\left|S_{3}\right| \leq 0.9$ which again shows that this sample is free of orientation bias. Note that my sample is not volume limited, a major drawback in such a study, nor is it redshift complete a fact that could hinder me from reaching any strong conclusion, especially if I would not detect any alignments.

The distance to each cluster is determined by the usual relation (Mattig 1958):

$$
R=\frac{c}{H_{\circ} q_{\circ}^{2}(1+z)^{2}}\left[q_{\circ} z+\left(q_{\circ}-1\right)\left[\left(1+2 q_{\circ} z\right)^{\frac{1}{2}}-1\right]\right]
$$

with $q_{\mathrm{o}}=0.2$. The cluster coordinate system is transformed to a Cartesian one using the transformations: $x=R \cos b \sin l, y=R \cos b \cos l$ and $z=R \sin b$. In this coordinate system the relative distance $D_{i j}$ of each cluster pair are found by $D_{i j}=\left(\delta x_{i j}^{2}+\delta y_{i j}^{2}+\delta z_{i j}^{2}\right)^{1 / 2}$. I determine the position angle $\phi_{i j}$ of each cluster, $i$, relative to the direction of a neighbour, $j$, as the acute angle between the major axis of the cluster and the great circle connecting the two clusters. Firstly the position angle of the cluster-pair separation, $\phi_{i s}$, at the position of the primary cluster is found by using straightforward spherical trigonometry and finally $\phi_{i j}=\theta_{i}-\phi_{i s}$, where $\theta_{i}$ is the position angle of the primary cluster.

The mean $\phi_{i j}$ in an isotropic distribution with large $N$ would be equal to $45^{\circ}$. A deviation from this value, in a bias free sample, would be an indication of an alignment/misalignment effect. A useful measure of such an effect is given by Struble \& Peebles (1985):

$$
\delta=\sum_{i=1}^{N} \frac{\phi_{i j}}{N}-45
$$

If the values of $\phi_{i j}$ are isotropically distributed between $0^{\circ}$ and $90^{\circ}$ then for large $N,\langle\delta\rangle=0$ and the standard deviation is:

$$
\sigma=\frac{90}{\sqrt{12 N}}
$$

A negative value of $\delta$ would indicate alignment and a positive one a misalignment. We must consider though that a comparison of observations with these formulas should take place only if the determination of position angles does not entail systematic errors. If there is a systematic bias of the cluster position angles towards a preferred direction it could produce a false alignment or misalignment signal. This, however, is not the case for my samples since it was found, in section 3 , that such a bias is not present. I searched for two types of cluster alignments:

1. Nearest-Neighbour alignments $(\mathrm{N}-\mathrm{N})$

2. All neighbour alignments within superclusters (A-N) 


\subsection{Nearest-Neighbour Alignments}

To study this type of alignments I found for each cluster $i$ a neighbour $j$ for which their distance is $\min \left[D_{i j}\right]$. For such pairs we then calculate $\phi_{i j}\left(\equiv \phi_{n n}\right)$ and estimate the alignment signal $\delta$ and its deviation $\sigma$. Note that $\mu \equiv\left\langle\min \left[D_{i j}\right]\right\rangle$ is $\sim 25 h^{-1} \mathrm{Mpc}$, a rather large value but consider that the sample is not volume limited and there are a few clusters with very large redshifts. If we limit the $\mathrm{N}-\mathrm{N}$ separations to a maximum value of 30 or $50 h^{-1} \mathrm{Mpc}$ then $\mu \sim 14$ and $20 h^{-1} \mathrm{Mpc}$, respectively.

In Table 2 we present our results for different limiting cluster pair separations, $D_{\text {lim }}$. A quite significant signal (2.5 $\sigma$ effect) is found when $D_{\text {lim }}=15 \mathrm{~h}^{-1} \mathrm{Mpc}$ which persists, although having a smaller value of $\delta$, even when very large separations are considered $\left(D_{\text {lim }}=50-60 h^{-1} \mathrm{Mpc}\right)$. Figure 3 shows the frequency distribution of the $\phi_{n n}$ angles. Figure $3 \mathrm{a}$ shows the results for $D_{\text {lim }}=10$ and $15 h^{-1} \mathrm{Mpc}$ (solid and dashed lines respectively) while figure $3 \mathrm{~b}$ shows the results when all the $\mathrm{N}-\mathrm{N}$ separations are considered. A clear excess of small $\phi_{n n}$ values is present in all cases, being more pronounced at smaller values of $D_{\text {lim }}$.

I attempted to further test the significance of my result by testing whether the observed $\phi_{n n}$ distribution could have been drawn from a uniform one. To this end we perform a KolmogorovSmirnov two-sided test and we find that the probability of the observed distribution being drawn from a uniform parent distribution is $\sim 4 \times 10^{-2}, \sim 3 \times 10^{-2}$ and $\sim 5 \times 10^{-2}$ for $D_{\text {lim }}=10,15$ and $60 h^{-1}$ Mpc respectively.

\subsection{All-Neighbour Alignments within Superclusters}

I have used the above procedure to study alignments between all possible cluster-pairs that lie within the same supercluster. Binggeli (1982) has claimed a positive alignment signal of this type, although at a low significance level. Similarly, West (1989b) has found that such alignments occur over scales of at least $30 h^{-1} \mathrm{Mpc}$ and maybe over even larger distances, while Plionis, Valdarnini \& Jing (1992), using Abell clusters and a subset of the position angles presented here found that indeed there is a strong alignment signal between all neighbours within superclusters which seems also to be correlated with the shape of the supercluster, being stronger in prolate configurations.

I define superclusters using a friend of friend algorithm and two percolation radii, $R_{p}$, of $35 h^{-1}$ and $25 h^{-1} \mathrm{Mpc}$ length. Since my main results seem to be insensitive to these two choices of $R_{p}$, I will present only those for the $R_{p}=35 h^{-1}$ case. For this value of $R_{p}$ I obtain 33 superclusters (with more than two members) containing 209 out of the 277 clusters. The alignment signal (eq. 5) is used but note that I used clusters that belong into superclusters of any membership number above unity, while Plionis et.al. (1992) used supercluster with more than 8 members (because they wanted to determine also their shapes).

In table $3 \mathrm{I}$ present the values of the alignment signal $\delta$ and its significance for different cluster separation ranges. Strong alignments occur in my sample only for $D_{l i m}=10 h^{-1}$ Mpc while weaker but still significant alignments are present even when $D_{\text {lim }} \sim 150 h^{-1} \mathrm{Mpc}$. Remember, however, that my samples are not volume limited nor redshift complete and therefore even such an alignment signal 
should be considered as an indication of a probably larger and more significant alignment effect. In figure $4 \mathrm{I}$ present the frequency distribution of the $\phi_{i j}$ angles at four cluster separation ranges. The strong alignment effect at the $D \leq 10 h^{-1} \mathrm{Mpc}$ range is evident. Our results are in agreement with those of West (1989b) and Plionis et.al. (1992) even more so if we take into account that our samples are not as complete, a fact that would tend wash-out any inherent alignment signal.

\subsection{Possible systematic biases}

The observed alignments could be artificial, in principle, resulting from the fact that clusters which are near in space are in many occasions also near on the sky and therefore their galaxy density envelopes could overlap in the angular projection. This could produce a directional bias when determining their position angles and thus a preferred orientation along the direction of a neighbour, in particular of the nearest neighbour. A manifestation of such an effect would be to find a stronger and more significant alignment signal between nearest neighbours in angular space rather than in real 3-D space. Since however, a number of clusters that are neighbours in real space are neighbours also in angular space some weak alignment signal should survive the projection. To test whether this effect is responsible for the alignment signal found, I searched for nearest neighbour alignments in my sample but using angular separations instead of spatial ones. I found for all separations a significantly weaker alignment signal. For example when the angular separations are $\leq 1.4^{\circ}$ (for which $\mathrm{I}$ have in total 66 separation, a value similar to that for $D \leq 10 h^{-1} \mathrm{Mpc}$ ) I obtain a weaker and less significant alignment signal $\left(\delta=-4.1 \pm 3.2\right.$ ) with a 0.15 probability of the distribution of $\phi_{n n}$ being drawn from a uniform one, confirming that the observed alignment signal in $3-\mathrm{D}$ is not caused by the above mentioned bias.

Furthermore, to test whether the grid effect, mentioned in section 3 , has induced the detected cluster alignments, I repeated the $\mathrm{N}-\mathrm{N}$ analysis but after having reshuffled the cluster position angles. I performed a 100 such reshufflings and I find, for all separations considered, $\langle\delta\rangle=0$ with a standard deviation equal to that obtained from eq. 6 , which indicates that the grid effect is not responsible for the detected cluster alignment signal.

\section{Conclusions}

I have presented a large number of new cluster position angles. In total 637 position angles were estimated. Using available redshifts for a subset of these clusters I found a strong alignment signal $(\sim 2.5-3 \sigma)$ between nearest cluster neighbours when their separations are $\leq 15 h^{-1} \mathrm{Mpc}$, as well as a stronger $(\sim 4 \sigma)$ signal between all neighbours residing in superclusters when separations $\leq 10$ $h^{-1} \mathrm{Mpc}$ are considered. A quite weak but significant alignment signal persists even when considering larger separations (up to $150 \mathrm{~h}^{-1} \mathrm{Mpc}$ ). 
Table 1a: Mean position angles (column 5) of Abell and Shectman clusters (identified at the 3.6 level). We present the number of overdensity levels used to estimate the position angles (column 3 ), the minimum and maximum number of $10^{\prime} \times 10^{\prime}$ cells, $N_{\text {cells }}$, defining the cluster sampling area [at the different overdensity levels] (column 4), while for the cases were the position angle $(\theta)$ was determined in 3 or more overdensity levels we list also $\sigma_{\theta}$ (column 6 ) and the median value (column 7 ) [in those cases where 4 levels were used we averge the two central $\theta$ values]. 


\begin{tabular}{|c|c|c|c|c|c|c|}
\hline Shectman & Abell & No of levels & $\min -\max N_{\text {cells }}$ & $\theta$ & $\sigma_{\theta}$ & $\operatorname{Med}[\theta]$ \\
\hline 1 & 724 & 1 & 6 & 177.2 & $\ldots$ & $\ldots$ \\
\hline 2 & 727 & 2 & $10-15$ & 65.8 & $\ldots$ & $\ldots$ \\
\hline 4 & - & 2 & $7-12$ & 80.4 & $\ldots$ & $\ldots$ \\
\hline 5 & 757 & 2 & $5-9$ & 79.3 & $\ldots$ & $\ldots$ \\
\hline 6 & - & 2 & $6-14$ & 36.5 & $\ldots$ & $\ldots$ \\
\hline 8 & 819 & 1 & 7 & 111.0 & $\ldots$ & $\ldots$ \\
\hline 9 & 858 & 1 & 7 & 96.1 & $\ldots$ & $\ldots$ \\
\hline 10 & - & 2 & $7-10$ & 103.1 & $\ldots$ & $\ldots$ \\
\hline 11 & 879 & 1 & 8 & 132.4 & $\ldots$ & $\ldots$ \\
\hline 12 & - & 1 & 6 & 166.1 & $\ldots$ & $\ldots$ \\
\hline 16 & 912 & 1 & 6 & 0.6 & $\ldots$ & $\ldots$ \\
\hline 18 & 921 & 2 & 5-9 & 118.1 & $\ldots$ & $\ldots$ \\
\hline 21 & 949 & 2 & $7-9$ & 40.5 & $\ldots$ & $\ldots$ \\
\hline 22 & - & 3 & $8-20$ & 165.9 & 14.6 & 157.5 \\
\hline 23 & 952 & 2 & $7-10$ & 119.8 & $\ldots$ & $\ldots$ \\
\hline 24 & - & 3 & $5-13$ & 115.4 & 5.7 & 114.4 \\
\hline 25 & - & 3 & $6-13$ & 19.4 & 19.2 & 20.1 \\
\hline 26 & 978 & 3 & $8-13$ & 169.3 & 3.5 & 167.7 \\
\hline 27 & - & 2 & 5-7 & 47.0 & $\ldots$ & $\ldots$ \\
\hline 28 & 986 & 2 & $5-8$ & 119.9 & $\ldots$ & $\ldots$ \\
\hline 30 & - & 2 & $7-11$ & 97.8 & $\ldots$ & $\ldots$ \\
\hline 31 & - & 2 & $8-15$ & 157.2 & $\ldots$ & $\ldots$ \\
\hline 33 & 993 & 3 & $9-27$ & 114.9 & 3.4 & 113.8 \\
\hline 35 & - & 3 & $6-19$ & 133.7 & 7.1 & 131.8 \\
\hline 37 & - & 2 & $6-14$ & 22.0 & $\ldots$ & $\ldots$ \\
\hline 38 & - & 1 & 6 & 109.0 & $\ldots$ & $\ldots$ \\
\hline 39 & 1020 & 4 & $10-40$ & 137.1 & 19.2 & 144.6 \\
\hline 42 & - & 2 & 5-28 & 102.5 & $\ldots$ & $\ldots$ \\
\hline 43 & - & 1 & 7 & 60.9 & $\ldots$ & $\ldots$ \\
\hline 45 & - & 1 & 6 & 44.5 & $\ldots$ & $\ldots$ \\
\hline 46 & 1033 & 1 & 8 & 43.9 & $\ldots$ & $\ldots$ \\
\hline 47 & 1035 & 4 & $5-84$ & 93.8 & 21.1 & 90.6 \\
\hline 48 & - & 2 & $5-10$ & 131.6 & $\ldots$ & $\ldots$ \\
\hline 50 & 1050 & 3 & $5-13$ & 76.6 & 6.5 & 74.2 \\
\hline
\end{tabular}




\begin{tabular}{|c|c|c|c|c|c|c|}
\hline Shectman & Abell & No of levels & min-max $N_{\text {cells }}$ & $\theta$ & $\sigma_{\theta}$ & $\operatorname{Med}[\theta]$ \\
\hline 52 & 1066 & 4 & $6-27$ & 9.6 & 24.0 & 6.7 \\
\hline 53 & 1067 & 1 & 8 & 45.0 & $\ldots$ & $\ldots$ \\
\hline 54 & - & 1 & 10 & 157.4 & $\ldots$ & $\ldots$ \\
\hline 55 & - & 2 & $6-10$ & 116.9 & $\ldots$ & $\ldots$ \\
\hline 57 & 1085 & 1 & 8 & 176.6 & $\ldots$ & $\ldots$ \\
\hline 59 & 1100 & 2 & $5-10$ & 101.4 & $\ldots$ & $\ldots$ \\
\hline 60 & - & 2 & $13-25$ & 155.2 & $\ldots$ & $\ldots$ \\
\hline 61 & - & 3 & $5-11$ & 102.2 & 6.4 & 103.6 \\
\hline 62 & - & 2 & $7-9$ & 119.7 & $\ldots$ & $\ldots$ \\
\hline 63 & 1126 & 1 & 5 & 161.8 & $\ldots$ & ... \\
\hline 65 & - & 2 & $6-11$ & 91.5 & $\ldots$ & ... \\
\hline 66 & 1139 & 2 & $6-14$ & 112.5 & $\ldots$ & $\ldots$ \\
\hline 67 & - & 1 & 10 & 44.5 & $\ldots$ & $\ldots$ \\
\hline 68 & 1149 & 2 & $7-9$ & 138.2 & $\ldots$ & $\ldots$ \\
\hline 69 & - & 2 & 6-9 & 61.5 & $\ldots$ & $\ldots$ \\
\hline 70 & - & 2 & 5-11 & 135.6 & $\ldots$ & $\ldots$ \\
\hline 71 & 1169 & 4 & $9-89$ & 52.1 & 33.8 & 57.5 \\
\hline 72 & 1168 & 2 & 5-11 & 44.0 & $\ldots$ & $\ldots$ \\
\hline 73 & 1173 & 2 & 5-9 & 102.5 & $\ldots$ & $\ldots$ \\
\hline 74 & 1177 & 2 & $8-16$ & 13.9 & $\ldots$ & $\ldots$ \\
\hline 75 & 1185 & 3 & $14-51$ & 169.4 & 47.4 & 145.4 \\
\hline 76 & - & 1 & 13 & 168.5 & $\ldots$ & $\ldots$ \\
\hline 77 & 1190 & 1 & 5 & 180.0 & $\ldots$ & $\ldots$ \\
\hline 78 & 1187 & 1 & 11 & 156.7 & $\ldots$ & $\ldots$ \\
\hline 82 & 1205 & 3 & $8-16$ & 89.3 & 7.4 & 91.4 \\
\hline 83 & - & 1 & 7 & 61.5 & $\ldots$ & $\ldots$ \\
\hline 84 & - & 1 & 9 & 21.3 & $\ldots$ & $\ldots$ \\
\hline 85 & - & 2 & $11-19$ & 54.3 & $\ldots$ & $\ldots$ \\
\hline 86 & 1213 & 3 & $12-29$ & 96.0 & 10.4 & 90.2 \\
\hline 87 & - & 1 & 5 & 57.6 & $\ldots$ & $\ldots$ \\
\hline 88 & - & 3 & $5-16$ & 116.0 & 49.4 & 107.1 \\
\hline 89 & - & 4 & $8-26$ & 50.8 & 29.0 & 43.2 \\
\hline 90 & - & 3 & $8-20$ & 6.4 & 53.5 & 2.5 \\
\hline 91 & 1235 & 2 & $10-11$ & 73.6 & $\ldots$ & $\ldots$ \\
\hline
\end{tabular}




\begin{tabular}{|c|c|c|c|c|c|c|}
\hline Shectman & Abell & No of levels & $\min -\max N_{\text {cells }}$ & $\theta$ & $\overline{\sigma_{\theta}}$ & $\operatorname{Med}[\theta]$ \\
\hline 97 & - & 1 & 6 & 30.8 & $\ldots$ & ... \\
\hline 99 & - & 1 & 8 & 135.0 & $\ldots$ & ... \\
\hline 100 & 1267 & 2 & $6-9$ & 93.3 & $\ldots$ & ... \\
\hline 102 & - & 1 & 7 & 7.7 & $\ldots$ & ... \\
\hline 103 & - & 2 & $8-13$ & 33.7 & $\ldots$ & ... \\
\hline 104 & - & 1 & 15 & 107.6 & $\ldots$ & ... \\
\hline 105 & 1291 & 3 & $7-15$ & 172.6 & 21.5 & 1.6 \\
\hline 106 & - & 2 & $5-7$ & 103.2 & $\ldots$ & ... \\
\hline 107 & 1307 & 3 & $5-12$ & 6.9 & 9.3 & 5.0 \\
\hline 108 & 1308 & 1 & 7 & 80.2 & $\ldots$ & ... \\
\hline 109 & - & 1 & 7 & 135.8 & $\ldots$ & $\ldots$ \\
\hline 110 & 1314 & 4 & $10-26$ & 75.0 & 2.0 & 74.4 \\
\hline 111 & - & 2 & $8-18$ & 97.2 & $\ldots$ & ... \\
\hline 112 & 1317 & 3 & $6-13$ & 42.6 & 38.2 & 21.2 \\
\hline 113 & - & 2 & $7-13$ & 85.2 & $\ldots$ & ... \\
\hline 114 & 1318 & 1 & 8 & 1.6 & $\ldots$ & ... \\
\hline 115 & - & 1 & 5 & 105.5 & $\ldots$ & ... \\
\hline 117 & - & 1 & 5 & 18.0 & $\ldots$ & ... \\
\hline 118 & 1332 & 1 & 7 & 135.8 & $\ldots$ & $\ldots$ \\
\hline 119 & 1336 & 2 & $8-11$ & 120.4 & $\ldots$ & $\ldots$ \\
\hline 121 & 1341 & 4 & $13-45$ & 159.6 & 20.9 & 160.1 \\
\hline 122 & 1346 & 2 & $17-26$ & 164.8 & $\ldots$ & ... \\
\hline 124 & 1364 & 1 & 12 & 120.9 & $\ldots$ & $\ldots$ \\
\hline 125 & 1362 & 3 & $9-26$ & 149.8 & 5.7 & 148.2 \\
\hline 126 & - & 1 & 7 & 78.3 & $\ldots$ & ... \\
\hline 131 & 1367 & 3 & $21-46$ & 156.8 & 20.9 & 150.6 \\
\hline 128 & - & 1 & 6 & 90.0 & $\ldots$ & ... \\
\hline 129 & - & 2 & $9-14$ & 118.2 & $\ldots$ & ... \\
\hline 130 & 1365 & 3 & $8-12$ & 11.7 & 12.4 & 7.3 \\
\hline 134 & - & 1 & 5 & 20.4 & $\ldots$ & ... \\
\hline 135 & 1371 & 2 & $8-17$ & 100.1 & $\ldots$ & ... \\
\hline 136 & - & 3 & $9-20$ & 126.5 & 1.4 & 126.4 \\
\hline 137 & 1377 & 3 & $10-19$ & 165.3 & 13.4 & 165.5 \\
\hline 138 & 1380 & 1 & 7 & 173.9 & $\ldots$ & ... \\
\hline
\end{tabular}




\begin{tabular}{|c|c|c|c|c|c|c|}
\hline Shectman & Abell & No of levels & $\min -\max N_{\text {cells }}$ & $\theta$ & $\sigma_{\theta}$ & $\operatorname{Med}[\theta]$ \\
\hline 139 & - & 1 & 5 & 17.4 & $\ldots$ & ... \\
\hline 140 & - & 1 & 5 & 72.2 & $\ldots$ & ... \\
\hline 142 & 1383 & 2 & $5-8$ & 78.9 & $\ldots$ & ... \\
\hline 144 & - & 2 & $8-12$ & 148.8 & $\ldots$ & $\ldots$ \\
\hline 145 & - & 1 & 6 & 90.0 & $\ldots$ & ... \\
\hline 148 & - & 3 & $5-17$ & 152.1 & 11.8 & 158.4 \\
\hline 151 & 1399 & 1 & 9 & 149.4 & $\ldots$ & ... \\
\hline 153 & - & 1 & 6 & 0.8 & $\ldots$ & $\ldots$ \\
\hline 154 & - & 2 & $5-9$ & 92.8 & $\ldots$ & ... \\
\hline 155 & - & 2 & $7-17$ & 135.0 & $\ldots$ & ... \\
\hline 156 & - & 1 & 9 & 117.1 & $\ldots$ & ... \\
\hline 157 & 1407 & 1 & 6 & 2.2 & $\ldots$ & $\ldots$ \\
\hline 158 & 1413 & 2 & $9-23$ & 107.0 & $\ldots$ & ... \\
\hline 159 & 1416 & 1 & 5 & 73.9 & $\ldots$ & $\ldots$ \\
\hline 161 & - & 3 & $5-13$ & 89.3 & 13.1 & 95.8 \\
\hline 162 & 1424 & 3 & $5-12$ & 57.0 & 9.8 & 58.6 \\
\hline 163 & 1436 & 3 & $10-14$ & 29.5 & 3.3 & 31.4 \\
\hline 164 & 1448 & 2 & $5-6$ & 121.3 & $\ldots$ & $\ldots$ \\
\hline 165 & 1452 & 1 & 5 & 109.4 & $\ldots$ & $\ldots$ \\
\hline 167 & - & 2 & 8-11 & 106.7 & $\ldots$ & $\ldots$ \\
\hline 169 & - & 2 & $5-7$ & 165.8 & $\ldots$ & $\ldots$ \\
\hline 170 & 1468 & 2 & 5-7 & 37.4 & $\ldots$ & $\ldots$ \\
\hline 171 & - & 3 & $9-32$ & 4.7 & 7.4 & 8.1 \\
\hline 174 & 1502 & 2 & $7-11$ & 7.1 & $\ldots$ & $\ldots$ \\
\hline 175 & - & 3 & $10-24$ & 143.6 & 15.8 & 138.0 \\
\hline 176 & 1507 & 3 & $7-10$ & 60.7 & 11.2 & 64.8 \\
\hline 177 & - & 1 & 7 & 60.3 & $\ldots$ & ... \\
\hline 178 & - & 3 & $8-18$ & 16.3 & 22.3 & 13.7 \\
\hline 180 & 1516 & 2 & $9-20$ & 89.6 & $\ldots$ & ... \\
\hline 181 & 1517 & 2 & $9-20$ & 158.0 & $\ldots$ & ... \\
\hline 183 & 1520 & 4 & $17-52$ & 8.6 & 19.0 & 0.5 \\
\hline 184 & - & 1 & 5 & 19.5 & $\ldots$ & $\ldots$ \\
\hline 185 & - & 3 & 5-13 & 173.6 & 45.7 & 162.8 \\
\hline 186 & 1535 & 3 & $7-13$ & 141.2 & 41.2 & 129.9 \\
\hline
\end{tabular}




\begin{tabular}{|c|c|c|c|c|c|c|}
\hline Shectman & Abell & No of levels & $\min -\max N_{\text {cells }}$ & $\theta$ & $\sigma_{\theta}$ & $\operatorname{Med}[\theta]$ \\
\hline 187 & 1541 & 4 & $8-44$ & 117.0 & 9.3 & 114.2 \\
\hline 189 & - & 2 & $6-14$ & 160.4 & $\ldots$ & ... \\
\hline 190 & 1552 & 4 & $5-40$ & 163.3 & 42.6 & 165.0 \\
\hline 192 & 1553 & 1 & 8 & 137.1 & $\cdots$ & ... \\
\hline 193 & 1555 & 3 & $6-20$ & 96.0 & 7.9 & 94.7 \\
\hline 194 & - & 2 & $5-12$ & 113.3 & $\ldots$ & ... \\
\hline 195 & 1564 & 2 & $5-10$ & 111.1 & $\ldots$ & ... \\
\hline 196 & 1569 & 3 & $7-18$ & 104.1 & 4.7 & 104.6 \\
\hline 197 & - & 1 & 5 & 162.0 & $\ldots$ & ... \\
\hline 198 & - & 1 & 15 & 148.6 & $\ldots$ & ... \\
\hline 199 & 1589 & 3 & $7-15$ & 142.0 & 34.5 & 152.9 \\
\hline 201 & - & 2 & $7-22$ & 142.9 & $\cdots$ & ... \\
\hline 203 & - & 1 & 8 & 177.8 & $\cdots$ & $\ldots$ \\
\hline 204 & 1620 & 3 & $8-23$ & 80.5 & 10.0 & 78.9 \\
\hline 208 & - & 2 & $7-14$ & 48.5 & $\ldots$ & $\ldots$ \\
\hline 209 & 1631 & 3 & $29-73$ & 142.5 & 5.7 & 142.6 \\
\hline 210 & - & 2 & $5-8$ & 120.6 & $\ldots$ & ... \\
\hline 211 & - & 1 & 6 & 64.1 & $\ldots$ & ... \\
\hline 212 & 1638 & 3 & 5-14 & 26.9 & 5.1 & 29.4 \\
\hline 213 & - & 3 & $5-10$ & 91.3 & 3.8 & 90.8 \\
\hline 215 & 1644 & 3 & $20-48$ & 86.4 & 42.8 & 91.8 \\
\hline 216 & - & 3 & $21-45$ & 128.8 & 1.2 & 128.1 \\
\hline 218 & - & 1 & 9 & 105.7 & $\ldots$ & ... \\
\hline 219 & 1650 & 4 & $6-17$ & 145.6 & 6.2 & 144.6 \\
\hline 221 & 1651 & 2 & $7-13$ & 59.3 & $\ldots$ & $\ldots$ \\
\hline 222 & 1656 & 3 & $32-67$ & 71.5 & 1.0 & 71.8 \\
\hline 224 & 1663 & 3 & $7-14$ & 30.7 & 20.0 & 39.0 \\
\hline 226 & - & 2 & $6-12$ & 73.8 & $\ldots$ & ... \\
\hline 227 & - & 3 & $6-46$ & 91.7 & 41.0 & 109.1 \\
\hline 228 & - & 2 & $5-13$ & 113.1 & $\ldots$ & ... \\
\hline 229 & 1668 & 1 & 6 & 48.4 & $\ldots$ & ... \\
\hline 230 & - & 2 & $5-7$ & 5.8 & $\ldots$ & ... \\
\hline 231 & - & 2 & $9-36$ & 101.1 & $\ldots$ & ... \\
\hline 232 & - & 2 & $6-12$ & 37.6 & $\ldots$ & $\ldots$ \\
\hline
\end{tabular}




\begin{tabular}{|c|c|c|c|c|c|c|}
\hline Shectman & Abell & No of levels & $\min -\max N_{\text {cells }}$ & $\theta$ & $\sigma_{\theta}$ & $\operatorname{Med}[\theta]$ \\
\hline 234 & 1691 & 3 & $14-26$ & 121.2 & 0.7 & 120.8 \\
\hline 235 & - & 3 & $6-14$ & 130.9 & 23.9 & 138.0 \\
\hline 236 & 1706 & 2 & $10-16$ & 121.8 & $\ldots$ & $\ldots$ \\
\hline 237 & - & 1 & 6 & 45.5 & $\ldots$ & $\ldots$ \\
\hline 238 & 1709 & 2 & $5-8$ & 158.0 & $\ldots$ & ... \\
\hline 240 & 1711 & 1 & 5 & 72.2 & $\ldots$ & $\ldots$ \\
\hline 241 & - & 4 & $6-75$ & 127.2 & 22.0 & 125.9 \\
\hline 243 & - & 1 & 9 & 158.9 & $\ldots$ & $\ldots$ \\
\hline 244 & - & 3 & $6-16$ & 106.9 & 25.7 & 104.8 \\
\hline 246 & - & 2 & $6-12$ & 22.5 & $\ldots$ & $\ldots$ \\
\hline 247 & - & 3 & $5-27$ & 69.2 & 18.9 & 71.4 \\
\hline 248 & 1738 & 3 & $5-17$ & 113.0 & 16.0 & 108.2 \\
\hline 249 & 1749 & 4 & $8-19$ & 125.1 & 9.6 & 122.4 \\
\hline 250 & - & 3 & $10-27$ & 168.2 & 13.6 & 169.4 \\
\hline 253 & - & 4 & $7-19$ & 79.7 & 22.2 & 90.0 \\
\hline 254 & - & 2 & $5-8$ & 35.5 & $\ldots$ & $\ldots$ \\
\hline 256 & - & 1 & 8 & 50.0 & $\ldots$ & $\ldots$ \\
\hline 257 & 1764 & 1 & 5 & 161.5 & $\cdots$ & $\cdots$ \\
\hline 258 & - & 1 & 9 & 20.8 & $\ldots$ & $\cdots$ \\
\hline 259 & 1767 & 1 & 8 & 33.4 & $\ldots$ & $\ldots$ \\
\hline 261 & - & 2 & $6-8$ & 132.8 & $\ldots$ & $\ldots$ \\
\hline 262 & - & 1 & 7 & 59.0 & $\ldots$ & $\ldots$ \\
\hline 263 & 1775 & 3 & $12-41$ & 88.3 & 6.0 & 90.9 \\
\hline 264 & 1773 & 3 & $5-14$ & 61.1 & 28.4 & 71.1 \\
\hline 265 & . & 3 & $11-44$ & 89.0 & 21.5 & 97.5 \\
\hline 266 & 1778 & 4 & $6-37$ & 86.8 & 12.1 & 84.2 \\
\hline 267 & 1783 & 4 & $6-18$ & 77.0 & 36.3 & 65.3 \\
\hline 268 & 1780 & 1 & 5 & 109.2 & $\ldots$ & $\ldots$ \\
\hline 270 & 1793 & 1 & 6 & 92.6 & $\ldots$ & $\ldots$ \\
\hline 271 & - & 2 & $8-10$ & 124.7 & $\ldots$ & $\ldots$ \\
\hline 272 & 1795 & 3 & $11-21$ & 13.8 & 12.6 & 16.5 \\
\hline 273 & 1800 & 3 & $9-18$ & 48.9 & 22.3 & 57.1 \\
\hline 274 & - & 2 & $6-12$ & 62.2 & $\ldots$ & $\ldots$ \\
\hline 275 & 1797 & 2 & $9-16$ & 135.4 & $\ldots$ & $\ldots$ \\
\hline
\end{tabular}




\begin{tabular}{|c|c|c|c|c|c|c|}
\hline Shectman & Abell & No of levels & $\min -\max N_{\text {cells }}$ & $\theta$ & $\sigma_{\theta}$ & $\operatorname{Med}[\theta]$ \\
\hline 276 & - & 1 & 6 & 179.2 & $\ldots$ & ... \\
\hline 277 & - & 3 & $6-25$ & 26.9 & 8.4 & 23.5 \\
\hline 278 & - & 2 & $6-9$ & 94.8 & $\ldots$ & $\ldots$ \\
\hline 279 & - & 3 & $5-14$ & 85.9 & 13.4 & 93.0 \\
\hline 280 & 1809 & 4 & $8-12$ & 83.8 & 6.5 & 86.8 \\
\hline 282 & - & 4 & $5-37$ & 22.0 & 10.8 & 21.1 \\
\hline 283 & 1812 & 3 & $5-11$ & 14.7 & 5.4 & 16.6 \\
\hline 285 & - & 3 & $6-28$ & 164.9 & 12.6 & 162.2 \\
\hline 286 & - & 1 & 5 & 158.9 & $\ldots$ & ... \\
\hline 287 & - & 2 & $6-10$ & 81.4 & $\ldots$ & ... \\
\hline 288 & 1825 & 1 & 5 & 161.8 & $\ldots$ & ... \\
\hline 289 & 1834 & 3 & $5-14$ & 157.2 & 18.1 & 161.6 \\
\hline 290 & 1831 & 4 & $11-74$ & 130.9 & 35.4 & 124.9 \\
\hline 291 & 1836 & 3 & $10-25$ & 18.3 & 15.9 & 25.0 \\
\hline 293 & 1852 & 3 & $6-15$ & 1.2 & 10.1 & $\ldots$ \\
\hline 294 & - & 4 & $6-35$ & 88.9 & 9.6 & 88.0 \\
\hline 296 & - & 1 & 8 & 70.7 & $\ldots$ & ... \\
\hline 297 & - & 1 & 6 & 4.3 & $\ldots$ & ... \\
\hline 298 & - & 1 & 6 & 91.6 & $\ldots$ & $\ldots$ \\
\hline 300 & 1873 & 1 & 8 & 178.5 & $\ldots$ & $\ldots$ \\
\hline 301 & - & 2 & $5-27$ & 171.9 & $\ldots$ & $\ldots$ \\
\hline 304 & - & 2 & $5-6$ & 29.4 & $\ldots$ & ... \\
\hline 305 & - & 1 & 6 & 91.5 & $\ldots$ & $\ldots$ \\
\hline 306 & 1882 & 2 & $8-16$ & 90.7 & $\ldots$ & $\ldots$ \\
\hline 307 & - & 2 & $7-14$ & 71.3 & $\ldots$ & ... \\
\hline 308 & 1890 & 3 & $8-11$ & 26.2 & 34.9 & 46.3 \\
\hline 309 & - & 3 & $11-18$ & 11.9 & 7.2 & 14.8 \\
\hline 310 & - & 2 & $5-12$ & 32.3 & $\ldots$ & ... \\
\hline 312 & - & 3 & $6-12$ & 61.1 & 15.9 & 53.0 \\
\hline 313 & - & 2 & $6-11$ & 31.9 & $\ldots$ & ... \\
\hline 314 & - & 3 & $9-14$ & 111.2 & 4.4 & 110.0 \\
\hline 315 & 1899 & 1 & 6 & 91.3 & $\ldots$ & ... \\
\hline 316 & 1904 & 4 & $7-20$ & 25.2 & 14.8 & 23.7 \\
\hline 317 & 1906 & 2 & $5-8$ & 63.3 & $\ldots$ & ... \\
\hline
\end{tabular}




\begin{tabular}{|c|c|c|c|c|c|c|}
\hline Shectman & Abell & No of levels & $\min -\max N_{\text {cells }}$ & $\theta$ & $\sigma_{\theta}$ & $\operatorname{Med}[\theta]$ \\
\hline 318 & 1908 & 2 & $11-18$ & 20.7 & $\ldots$ & $\ldots$ \\
\hline 319 & - & 1 & 6 & 88.4 & $\ldots$ & $\ldots$ \\
\hline 321 & 1913 & 4 & $14-91$ & 141.9 & 26.9 & 132.8 \\
\hline 322 & - & 1 & 5 & 69.7 & $\ldots$ & $\ldots$ \\
\hline 323 & - & 2 & $5-7$ & 10.0 & $\ldots$ & ... \\
\hline 324 & - & 1 & 12 & 101.0 & $\ldots$ & $\ldots$ \\
\hline 327 & - & 3 & $5-20$ & 99.8 & 8.5 & 96.9 \\
\hline 329 & - & 1 & 6 & 59.0 & $\ldots$ & $\ldots$ \\
\hline 331 & - & 3 & $5-15$ & 21.2 & 6.1 & 18.5 \\
\hline 332 & - & 3 & $5-16$ & 58.3 & 42.5 & 70.6 \\
\hline 333 & 1964 & 4 & $5-13$ & 53.3 & 13.4 & 49.3 \\
\hline 334 & - & 1 & 9 & 108.6 & $\ldots$ & $\ldots$ \\
\hline 335 & - & 1 & 7 & 81.7 & $\ldots$ & $\ldots$ \\
\hline 339 & 1983 & 3 & $11-24$ & 157.4 & 7.1 & 154.6 \\
\hline 340 & 1986 & 1 & 5 & 20.8 & $\ldots$ & $\ldots$ \\
\hline 341 & 1991 & 4 & $9-44$ & 5.0 & 25.8 & 4.5 \\
\hline 342 & - & 2 & $6-9$ & 50.5 & $\ldots$ & $\ldots$ \\
\hline 343 & - & 2 & $5-8$ & 120.3 & $\cdots$ & $\ldots$ \\
\hline 345 & 2020 & 3 & $5-13$ & 17.6 & 8.7 & 18.8 \\
\hline 346 & - & 1 & 5 & 161.9 & $\ldots$ & $\ldots$ \\
\hline 347 & 2022 & 4 & $7-34$ & 112.3 & 22.1 & 112.1 \\
\hline 348 & - & 2 & $8-10$ & 53.3 & $\ldots$ & $\ldots$ \\
\hline 350 & - & 1 & 5 & 20.1 & $\ldots$ & $\ldots$ \\
\hline 351 & - & 1 & 15 & 136.5 & $\ldots$ & ... \\
\hline 352 & - & 2 & $7-14$ & 19.1 & $\ldots$ & $\ldots$ \\
\hline 353 & 2028 & 3 & $5-37$ & 93.4 & 19.4 & 103.7 \\
\hline 354 & 2029 & 1 & 23 & 5.1 & $\ldots$ & $\ldots$ \\
\hline 362 & - & 3 & $10-19$ & 124.6 & 3.7 & 126.4 \\
\hline 365 & 2048 & 1 & 45 & 106.2 & $\ldots$ & $\ldots$ \\
\hline 366 & 2052 & 2 & $8-12$ & 46.4 & $\ldots$ & $\ldots$ \\
\hline 368 & - & 1 & 9 & 3.0 & $\ldots$ & $\ldots$ \\
\hline 369 & 2055 & 1 & 6 & 139.4 & $\ldots$ & $\ldots$ \\
\hline 370 & - & 3 & $5-34$ & 89.9 & 21.6 & 83.8 \\
\hline 371 & - & 1 & 31 & 138.4 & $\ldots$ & $\ldots$ \\
\hline
\end{tabular}




\begin{tabular}{|c|c|c|c|c|c|c|}
\hline Shectman & Abell & No of levels & $\min -\max N_{c e l l s}$ & $\theta$ & $\sigma_{\theta}$ & $\operatorname{Med}[\theta]$ \\
\hline 372 & - & 1 & 6 & 137.1 & $\ldots$ & ... \\
\hline 374 & - & 1 & 8 & 135.5 & $\ldots$ & ... \\
\hline 375 & - & 1 & 8 & 90.5 & $\ldots$ & ... \\
\hline 376 & 2062 & 3 & $5-8$ & 83.9 & 44.0 & 72.1 \\
\hline 377 & 2061 & 3 & $20-39$ & 35.2 & 6.7 & 32.8 \\
\hline 378 & 2063 & 1 & 9 & 45.1 & $\ldots$ & ... \\
\hline 380 & 2065 & 2 & $25-39$ & 176.1 & $\ldots$ & ... \\
\hline 383 & 2069 & 1 & 16 & 116.4 & $\ldots$ & $\ldots$ \\
\hline 384 & - & 1 & 6 & 89.5 & $\ldots$ & $\ldots$ \\
\hline 385 & 2079 & 1 & 10 & 37.0 & $\ldots$ & $\ldots$ \\
\hline 386 & 2083 & 4 & $6-20$ & 39.9 & 2.8 & 39.4 \\
\hline 389 & - & 3 & $6-22$ & 17.9 & 15.3 & 23.4 \\
\hline 390 & 2089 & 1 & 6 & 87.8 & $\ldots$ & $\ldots$ \\
\hline 391 & 2092 & 3 & $8-29$ & 24.9 & 18.8 & 32.9 \\
\hline 392 & 2107 & 3 & $7-14$ & 52.4 & 24.0 & 46.5 \\
\hline 393 & 2122 & 4 & $5-20$ & 150.9 & 16.1 & 156.2 \\
\hline 395 & - & 2 & $6-7$ & 124.7 & $\ldots$ & $\ldots$ \\
\hline 396 & - & 2 & $5-10$ & 177.7 & $\ldots$ & $\ldots$ \\
\hline 397 & - & 3 & $6-16$ & 90.8 & 2.9 & 89.4 \\
\hline 398 & - & 1 & 11 & 13.8 & $\ldots$ & $\ldots$ \\
\hline 400 & 2142 & 2 & $14-25$ & 145.1 & $\ldots$ & $\ldots$ \\
\hline 401 & - & 2 & $7-17$ & 139.1 & $\ldots$ & ... \\
\hline 403 & 2149 & 2 & $5-8$ & 111.4 & $\ldots$ & $\ldots$ \\
\hline 404 & - & 3 & $46-74$ & 126.7 & 25.1 & 112.9 \\
\hline 407 & 2151 & 3 & $21-34$ & 169.4 & 29.8 & 3.6 \\
\hline 409 & 2162 & 3 & $8-17$ & 1.7 & 10.2 & 7.3 \\
\hline 411 & - & 4 & $7-23$ & 82.2 & 8.3 & 83.3 \\
\hline 414 & - & 3 & $7-22$ & 54.1 & 46.5 & 72.0 \\
\hline 415 & - & 1 & 8 & 124.6 & $\ldots$ & $\ldots$ \\
\hline 416 & 2199 & 1 & 17 & 47.2 & $\ldots$ & $\ldots$ \\
\hline 417 & - & 1 & 8 & 124.6 & $\ldots$ & $\ldots$ \\
\hline 418 & - & 3 & $5-14$ & 68.8 & 10.4 & 69.7 \\
\hline 427 & - & 2 & $5-10$ & 41.9 & $\ldots$ & $\ldots$ \\
\hline 428 & - & 2 & 5-11 & 72.2 & $\ldots$ & $\ldots$ \\
\hline
\end{tabular}




\begin{tabular}{|c|c|c|c|c|c|c|}
\hline Shectman & Abell & No of levels & $\min -\max N_{\text {cells }}$ & $\theta$ & $\sigma_{\theta}$ & $\operatorname{Med}[\theta]$ \\
\hline 429 & 2366 & 2 & 8 & 133.6 & $\ldots$ & $\ldots$ \\
\hline 431 & 2372 & 2 & $5-6$ & 32.8 & $\ldots$ & $\ldots$ \\
\hline 432 & 2377 & 4 & $14-41$ & 122.7 & 14.7 & 127.2 \\
\hline 434 & 2382 & 2 & $7-9$ & 33.9 & $\ldots$ & $\ldots$ \\
\hline 435 & 2384 & 3 & $6-10$ & 132.3 & 39.3 & 126.9 \\
\hline 437 & - & 1 & 6 & 142.1 & $\ldots$ & ... \\
\hline 438 & - & 1 & 5 & 0.1 & $\ldots$ & $\ldots$ \\
\hline 439 & 2399 & 3 & $16-23$ & 94.4 & 9.2 & 99.6 \\
\hline 440 & 2401 & 4 & $5-18$ & 138.8 & 19.6 & 144.0 \\
\hline 441 & 2410 & 3 & $11-22$ & 121.9 & 4.1 & 123.9 \\
\hline 443 & 2415 & 2 & $7-11$ & 46.8 & $\ldots$ & $\ldots$ \\
\hline 444 & - & 2 & $6-7$ & 85.5 & $\ldots$ & $\ldots$ \\
\hline 445 & 2420 & 2 & $6-9$ & 59.7 & $\ldots$ & $\ldots$ \\
\hline 447 & 2426 & 1 & 6 & 23.8 & $\ldots$ & $\ldots$ \\
\hline 450 & - & 1 & 15 & 37.9 & $\ldots$ & $\ldots$ \\
\hline 452 & 2448 & 1 & 7 & 59.6 & $\ldots$ & $\ldots$ \\
\hline 453 & 2457 & 3 & $7-14$ & 25.5 & 3.6 & 25.3 \\
\hline 454 & 2459 & 4 & $7-21$ & 144.5 & 5.5 & 145.1 \\
\hline 456 & - & 1 & 6 & 134.2 & $\ldots$ & $\ldots$ \\
\hline 457 & - & 1 & 11 & 61.1 & $\ldots$ & ... \\
\hline 461 & - & 1 & 8 & 34.6 & $\ldots$ & $\ldots$ \\
\hline 463 & - & 3 & $5-20$ & 90.2 & 1.3 & 90.0 \\
\hline 464 & 2529 & 1 & 5 & 16.7 & $\ldots$ & ... \\
\hline 467 & - & 4 & $8-15$ & 52.3 & 17.1 & 43.7 \\
\hline 470 & 2554 & 3 & $5-13$ & 110.0 & 4.6 & 112.5 \\
\hline 472 & - & 2 & $8-10$ & 66.8 & $\ldots$ & $\ldots$ \\
\hline 473 & - & 3 & $8-19$ & 148.6 & 4.8 & 149.2 \\
\hline 475 & 2569 & 1 & 9 & 59.1 & $\ldots$ & $\ldots$ \\
\hline 478 & 2593 & 3 & $10-26$ & 179.0 & 4.4 & 178.5 \\
\hline 479 & 2592 & 2 & $6-7$ & 74.6 & $\ldots$ & $\ldots$ \\
\hline 486 & 2657 & 3 & $5-16$ & 84.7 & 14.6 & 82.9 \\
\hline 487 & - & 3 & 5-11 & 130.3 & 17.5 & 135.6 \\
\hline 488 & - & 2 & $5-8$ & 21.5 & $\ldots$ & $\ldots$ \\
\hline 490 & - & 1 & 6 & 0.1 & $\ldots$ & $\ldots$ \\
\hline
\end{tabular}




\begin{tabular}{|c|c|c|c|c|c|c|}
\hline Shectman & Abell & No of levels & $\min -\max N_{\text {cells }}$ & $\theta$ & $\sigma_{\theta}$ & $\operatorname{Med}[\theta]$ \\
\hline 491 & 2670 & 3 & $8-12$ & 33.2 & 4.9 & 32.7 \\
\hline 492 & - & 4 & $8-36$ & 39.1 & 12.8 & 37.2 \\
\hline 494 & - & 3 & $7-19$ & 178.1 & 18.4 & 3.0 \\
\hline 495 & - & 1 & 8 & 92.9 & $\ldots$ & ... \\
\hline 496 & 2686 & 3 & $8-22$ & 124.9 & 11.2 & 124.6 \\
\hline 498 & - & 3 & $6-12$ & 151.8 & 25.1 & 152.9 \\
\hline 500 & 13 & 3 & $7-14$ & 83.1 & 28.9 & 89.2 \\
\hline 502 & 16 & 3 & $5-10$ & 49.6 & 41.7 & 70.1 \\
\hline 503 & - & 3 & $5-11$ & 149.6 & 38.4 & 163.3 \\
\hline 504 & - & 3 & $8-22$ & 133.3 & 11.9 & 135.7 \\
\hline 505 & - & 1 & 5 & 70.4 & $\ldots$ & ... \\
\hline 508 & 23 & 2 & $14-19$ & 63.5 & $\ldots$ & ... \\
\hline 510 & - & 2 & $5-9$ & 145.2 & $\ldots$ & $\ldots$ \\
\hline 511 & 27 & 4 & $5-40$ & 65.2 & 39.2 & 48.6 \\
\hline 512 & - & 4 & $7-34$ & 131.0 & 38.7 & 134.9 \\
\hline 513 & 44 & 4 & $6-20$ & 153.0 & 18.2 & 147.8 \\
\hline 514 & - & 1 & 8 & 31.6 & $\ldots$ & $\ldots$ \\
\hline 517 & - & 2 & 5-11 & 54.1 & $\ldots$ & $\ldots$ \\
\hline 518 & - & 3 & $18-44$ & 165.8 & 2.6 & 166.1 \\
\hline 520 & - & 2 & $7-14$ & 145.6 & $\ldots$ & $\ldots$ \\
\hline 522 & - & 2 & $5-10$ & 100.2 & $\ldots$ & $\ldots$ \\
\hline 524 & 76 & 4 & $5-23$ & 17.9 & 14.9 & 20.5 \\
\hline 525 & 84 & 1 & 6 & 133.3 & $\ldots$ & ... \\
\hline 526 & 85 & 4 & $12-34$ & 163.1 & 4.3 & 162.9 \\
\hline 529 & - & 1 & 6 & 42.2 & $\ldots$ & ... \\
\hline 530 & 93 & 1 & 5 & 161.0 & $\ldots$ & $\ldots$ \\
\hline 532 & - & 1 & 12 & 18.2 & $\ldots$ & $\ldots$ \\
\hline 533 & 95 & 2 & $10-20$ & 45.7 & $\ldots$ & ... \\
\hline 534 & 98 & 4 & $6-11$ & 3.3 & 34.5 & 6.4 \\
\hline 536 & 112 & 1 & 9 & 53.6 & $\ldots$ & ... \\
\hline 539 & - & 2 & $6-9$ & 124.8 & $\ldots$ & ... \\
\hline 541 & - & 1 & 6 & 46.0 & $\ldots$ & ... \\
\hline 542 & - & 1 & 6 & 2.6 & $\ldots$ & ... \\
\hline 543 & 114 & 1 & 6 & 84.6 & $\ldots$ & $\ldots$ \\
\hline
\end{tabular}




\begin{tabular}{|c|c|c|c|c|c|c|}
\hline Shectman & Abell & No of levels & $\min -\max N_{\text {cells }}$ & $\theta$ & $\sigma_{\theta}$ & $\operatorname{Med}[\theta]$ \\
\hline 544 & - & 1 & 12 & 131.8 & $\ldots$ & ... \\
\hline 546 & - & 2 & $10-13$ & 40.8 & $\ldots$ & ... \\
\hline 547 & - & 2 & $5-10$ & 36.9 & $\ldots$ & ... \\
\hline 548 & 116 & 3 & $6-28$ & 145.9 & 44.0 & 161.2 \\
\hline 549 & 117 & 4 & $15-84$ & 35.2 & 17.1 & 32.6 \\
\hline 550 & 119 & 3 & $22-45$ & 28.3 & 1.0 & 28.8 \\
\hline 551 & - & 1 & 6 & 12.1 & $\ldots$ & ... \\
\hline 552 & 120 & 1 & 8 & 66.4 & $\ldots$ & ... \\
\hline 553 & - & 2 & $6-12$ & 15.4 & $\ldots$ & ... \\
\hline 555 & - & 3 & $5-14$ & 97.3 & 10.3 & 95.4 \\
\hline 557 & 126 & 2 & $5-12$ & 12.6 & $\ldots$ & ... \\
\hline 559 & - & 1 & 12 & 15.1 & $\ldots$ & $\ldots$ \\
\hline 560 & - & 1 & 5 & 81.9 & $\ldots$ & ... \\
\hline 561 & - & 1 & 17 & 47.1 & $\ldots$ & ... \\
\hline 563 & - & 1 & 12 & 150.6 & $\ldots$ & ... \\
\hline 565 & - & 3 & $7-28$ & 94.2 & 34.3 & 83.3 \\
\hline 567 & - & 2 & $7-22$ & 87.7 & $\ldots$ & $\ldots$ \\
\hline 568 & - & 2 & $7-12$ & 77.2 & $\ldots$ & $\ldots$ \\
\hline 569 & 147 & 4 & $10-23$ & 78.8 & 6.3 & 77.1 \\
\hline 570 & 150 & 3 & $5-10$ & 164.2 & 24.1 & 176.7 \\
\hline 571 & 151 & 3 & $29-47$ & 40.5 & 13.3 & 47.6 \\
\hline 572 & 154 & 1 & 9 & 172.2 & $\ldots$ & $\ldots$ \\
\hline 576 & 160 & 2 & $6-9$ & 174.1 & $\ldots$ & ... \\
\hline 578 & - & 1 & 10 & 0.8 & $\ldots$ & $\ldots$ \\
\hline 580 & - & 2 & $7-9$ & 22.8 & $\ldots$ & $\ldots$ \\
\hline 581 & 168 & 2 & $13-19$ & 149.1 & $\ldots$ & $\ldots$ \\
\hline 582 & 171 & 1 & 5 & 95.0 & $\ldots$ & ... \\
\hline 583 & - & 2 & $9-13$ & 177.3 & $\ldots$ & ... \\
\hline 584 & - & 1 & 8 & 35.3 & $\ldots$ & ... \\
\hline 585 & 175 & 2 & $6-19$ & 78.8 & $\ldots$ & ... \\
\hline 586 & - & 2 & $5-13$ & 95.9 & $\ldots$ & ... \\
\hline 587 & - & 3 & $7-14$ & 95.3 & 16.4 & 87.1 \\
\hline 588 & - & 2 & $6-14$ & 104.8 & $\ldots$ & ... \\
\hline 590 & 193 & 3 & $9-14$ & 80.1 & 8.4 & 82.5 \\
\hline
\end{tabular}




\begin{tabular}{|c|c|c|c|c|c|c|}
\hline Shectman & Abell & No of levels & $\min -\max N_{\text {cells }}$ & $\theta$ & $\sigma_{\theta}$ & $\operatorname{Med}[\theta]$ \\
\hline 591 & - & 3 & $9-31$ & 59.7 & 5.1 & 56.8 \\
\hline 592 & 194 & 3 & $6-22$ & 83.8 & 28.3 & 86.7 \\
\hline 593 & - & 3 & $9-37$ & 25.6 & 24.8 & 12.4 \\
\hline 594 & - & 3 & $5-18$ & 64.7 & 47.2 & 63.8 \\
\hline 596 & - & 1 & 7 & 68.7 & $\ldots$ & ... \\
\hline 598 & - & 3 & $5-29$ & 67.8 & 22.0 & 56.3 \\
\hline 599 & - & 1 & 13 & 110.4 & $\ldots$ & ... \\
\hline 600 & - & 1 & 5 & 20.4 & $\ldots$ & ... \\
\hline 601 & - & 3 & $7-20$ & 170.7 & 29.5 & 174.6 \\
\hline 603 & - & 3 & $8-51$ & 52.0 & 29.3 & 66.9 \\
\hline 604 & - & 2 & $5-8$ & 100.3 & $\ldots$ & ... \\
\hline 608 & 256 & 1 & 5 & 134.3 & $\ldots$ & $\ldots$ \\
\hline 609 & 257 & 3 & $5-17$ & 163.2 & 41.4 & 173.5 \\
\hline 612 & 274 & 3 & $6-12$ & 73.9 & 2.1 & 72.7 \\
\hline 615 & - & 2 & $8-13$ & 89.9 & $\ldots$ & $\ldots$ \\
\hline 616 & 295 & 3 & $6-31$ & 53.9 & 1.5 & 54.1 \\
\hline 618 & - & 3 & $5-63$ & 144.4 & 13.7 & 139.1 \\
\hline 619 & - & 1 & 5 & 74.9 & $\ldots$ & ... \\
\hline 620 & - & 2 & $6-11$ & 46.2 & $\ldots$ & $\ldots$ \\
\hline 622 & - & 3 & $6-12$ & 35.1 & 5.8 & 38.1 \\
\hline 623 & - & 1 & 6 & 12.4 & $\ldots$ & $\ldots$ \\
\hline 624 & - & 1 & 6 & 132.2 & $\ldots$ & $\ldots$ \\
\hline 625 & - & 3 & $8-21$ & 121.4 & 13.1 & 123.6 \\
\hline 627 & 367 & 3 & $7-11$ & 43.8 & 27.7 & 44.8 \\
\hline 628 & - & 2 & $5-7$ & 59.1 & $\ldots$ & $\ldots$ \\
\hline 629 & - & 2 & $11-17$ & 139.6 & $\ldots$ & ... \\
\hline 630 & - & 3 & $5-14$ & 12.6 & 30.4 & 12.7 \\
\hline 631 & - & 1 & 6 & 135.4 & $\ldots$ & ... \\
\hline 632 & - & 2 & $6-10$ & 67.0 & $\ldots$ & ... \\
\hline 633 & 400 & 4 & $5-42$ & 90.1 & 13.1 & 96.1 \\
\hline 635 & - & 1 & 10 & 56.8 & $\ldots$ & ... \\
\hline 638 & - & 3 & $6-21$ & 1.7 & 2.3 & 0.5 \\
\hline 639 & 415 & 3 & $6-9$ & 48.7 & 15.7 & 43.7 \\
\hline 640 & - & 3 & $10-27$ & 95.3 & 10.8 & 96.8 \\
\hline
\end{tabular}




\begin{tabular}{ccccrrr}
\hline \hline Shectman & Abell & No of levels & $\min -\max N_{\text {cells }}$ & $\theta$ & $\sigma_{\theta}$ & $\operatorname{Med}[\theta]$ \\
\hline & & & & & & \\
641 & 420 & 1 & 6 & 134.6 & $\ldots$ & $\ldots$ \\
642 & 423 & 1 & 6 & 91.7 & $\ldots$ & $\ldots$ \\
646 & - & 2 & $6-8$ & 43.3 & $\ldots$ & $\ldots$ \\
647 & - & 2 & $5-11$ & 74.1 & $\ldots$ & $\ldots$ \\
648 & - & 1 & 8 & 159.1 & $\ldots$ & $\ldots$ \\
649 & - & 2 & $5-10$ & 131.8 & $\ldots$ & $\ldots$
\end{tabular}


Table 1b: Position angles of Abell clusters, identified at the 3.6 level but estimated at the 2.5 level.

\begin{tabular}{crrrrrrrr}
\hline \hline Abell & $N_{\text {cells }}$ & $\theta$ & Abell & $N_{\text {cells }}$ & $\theta$ & Abell & $N_{\text {cells }}$ & $\theta$ \\
\hline & & & & & & & & \\
779 & 5 & 72.2 & 1518 & 5 & 69.9 & 148 & 8 & 56.2 \\
1069 & 8 & 138.7 & 1524 & 10 & 8.8 & 152 & 10 & 110.8 \\
1170 & 5 & 158.7 & 1729 & 14 & 50.5 & 225 & 5 & 72.2 \\
1174 & 6 & 147.5 & 1796 & 5 & 109.1 & 403 & 5 & 69.6 \\
1189 & 6 & 167.3 & 1818 & 10 & 107.5 & 2400 & 9 & 142.9 \\
1227 & 9 & 145.0 & 1921 & 8 & 179.9 & 2412 & 5 & 89.7 \\
1278 & 5 & 118.9 & 1926 & 6 & 47.9 & 2421 & 6 & 1.3 \\
1344 & 10 & 127.8 & 1953 & 14 & 111.0 & 2490 & 6 & 18.2 \\
1411 & 5 & 44.0 & 1982 & 13 & 59.9 & 2525 & 5 & 17.0 \\
1427 & 5 & 160.4 & 2120 & 5 & 18.1 & 2665 & 5 & 108.6 \\
1515 & 15 & 161.8 & 2153 & 6 & 119.6 & & & \\
\hline
\end{tabular}

Table 1c: Position angles of Abell clusters, identified at the 3.6 level but estimated at the 1.8 level.

\begin{tabular}{rrrrrrrrr}
\hline \hline Abell & $N_{\text {cells }}$ & $\theta$ & Abell & $N_{\text {cells }}$ & $\theta$ & Abell & $N_{\text {cells }}$ & $\theta$ \\
\hline & & & & & & & & \\
865 & 6 & 12.5 & 1741 & 5 & 109.3 & 2208 & 5 & 20.0 \\
903 & 33 & 117.5 & 1768 & 7 & 133.6 & 65 & 10 & 87.0 \\
985 & 11 & 105.5 & 1845 & 7 & 35.5 & 94 & 6 & 71.4 \\
987 & 7 & 171.6 & 1861 & 5 & 18.7 & 144 & 5 & 18.0 \\
1092 & 5 & 109.0 & 1870 & 10 & 72.1 & 211 & 8 & 163.6 \\
1201 & 23 & 166.8 & 1936 & 6 & 18.9 & 212 & 5 & 84.7 \\
1242 & 6 & 17.9 & 1956 & 6 & 133.6 & 240 & 9 & 36.0 \\
1262 & 5 & 71.1 & 1990 & 5 & 70.2 & 243 & 5 & 160.8 \\
1292 & 7 & 177.4 & 2021 & 6 & 13.1 & 2403 & 11 & 36.4 \\
1327 & 5 & 146.3 & 2025 & 7 & 83.1 & 2477 & 6 & 91.5 \\
1387 & 8 & 51.7 & 2100 & 6 & 14.2 & 2638 & 8 & 32.0 \\
1441 & 9 & 153.0 & 2106 & 6 & 93.6 & 2676 & 8 & 157.7 \\
1625 & 8 & 150.2 & 2195 & 13 & 85.6 & 2703 & 5 & 71.2 \\
1688 & 5 & 89.7 & 2196 & 5 & 70.6 & & & \\
\hline
\end{tabular}


Table 1d: Position angles of Abell clusters, identified at the 2.5 level but estimated at the 1.8 level. Note that there are 12 clusters in common with table $1 \mathrm{~b}$, which for comparison reasons we have not averaged their estimated position angles. 


\begin{tabular}{|c|c|c|c|c|c|c|c|c|}
\hline Abell & $N_{\text {cells }}$ & $\theta$ & Abell & $N_{\text {cells }}$ & $\theta$ & Abell & $N_{\text {cells }}$ & $\theta$ \\
\hline 716 & 5 & 69.8 & 1444 & 5 & 71.6 & 2148 & 8 & 90.5 \\
\hline 733 & 6 & 91.0 & 1466 & 8 & 115.2 & 2169 & 16 & 50.2 \\
\hline 779 & 13 & 77.6 & 1474 & 9 & 67.2 & 2175 & 9 & 103.9 \\
\hline 795 & 6 & 129.4 & 1495 & 21 & 144.9 & 2177 & 13 & 53.2 \\
\hline 803 & 8 & 47.7 & 1518 & 10 & 90.8 & 48 & 6 & 130.0 \\
\hline 866 & 7 & 82.3 & 1526 & 8 & 138.7 & 53 & 10 & 119.6 \\
\hline 878 & 17 & 97.1 & 1534 & 9 & 12.6 & 103 & 10 & 72.3 \\
\hline 929 & 9 & 84.8 & 1573 & 9 & 119.9 & 111 & 10 & 3.3 \\
\hline 967 & 6 & 109.1 & 1581 & 5 & 163.1 & 13 & 6 & 145.3 \\
\hline 992 & 370 & 170.8 & 1583 & 5 & 162.4 & 172 & 15 & 87.0 \\
\hline 1003 & 17 & 119.8 & 1595 & 14 & 22.3 & 179 & 12 & 136.2 \\
\hline 1022 & 11 & 58.4 & 1599 & 7 & 177.2 & 195 & 5 & 159.9 \\
\hline 1051 & 6 & 90.4 & 1606 & 10 & 78.7 & 207 & 8 & 12.1 \\
\hline 1069 & 12 & 179.1 & 1616 & 8 & 131.5 & 225 & 21 & 41.5 \\
\hline 1097 & 11 & 86.1 & 1630 & 5 & 160.1 & 229 & 8 & 22.4 \\
\hline 1098 & 16 & 121.3 & 1684 & 5 & 19.2 & 267 & 26 & 94.7 \\
\hline 1108 & 10 & 48.1 & 1690 & 9 & 101.2 & 292 & 7 & 84.5 \\
\hline 1109 & 30 & 7.1 & 1693 & 11 & 155.7 & 358 & 10 & 9.7 \\
\hline 1118 & 6 & 40.5 & 1696 & 11 & 6.3 & 395 & 16 & 19.6 \\
\hline 1132 & 10 & 110.2 & 1715 & 5 & 162.2 & 403 & 12 & 59.5 \\
\hline 1135 & 14 & 44.9 & 1729 & 31 & 47.9 & 410 & 6 & 91.9 \\
\hline 1141 & 6 & 88.1 & 1769 & 15 & 75.3 & 428 & 11 & 123.4 \\
\hline 1143 & 42 & 13.4 & 1808 & 6 & 88.4 & 2346 & 7 & 45.6 \\
\hline 1152 & 7 & 58.7 & 1823 & 6 & 42.8 & 2361 & 6 & 87.1 \\
\hline 1170 & 11 & 162.2 & 1844 & 14 & 12.7 & 2365 & 6 & 46.2 \\
\hline 1179 & 11 & 49.1 & 1850 & 18 & 54.4 & 2412 & 13 & 117.0 \\
\hline 1189 & 32 & 10.9 & 1860 & 8 & 134.7 & 2490 & 13 & 16.0 \\
\hline 1198 & 11 & 164.3 & 1891 & 6 & 133.5 & 2495 & 6 & 42.5 \\
\hline 1216 & 7 & 179.4 & 1909 & 7 & 80.8 & 2511 & 7 & 136.9 \\
\hline 1218 & 9 & 175.4 & 1944 & 13 & 42.7 & 2525 & 12 & 144.5 \\
\hline 1257 & 12 & 25.0 & 1960 & 16 & 108.9 & 2528 & 10 & 116.8 \\
\hline 1264 & 5 & 74.7 & 1976 & 7 & 46.8 & 2549 & 20 & 165.6 \\
\hline 1375 & 5 & 0.7 & 1999 & 9 & 21.0 & 2583 & 13 & 11.7 \\
\hline 1390 & 5 & 95.4 & 2019 & 8 & 170.8 & 2589 & 11 & 14.5 \\
\hline
\end{tabular}




\begin{tabular}{rrrrrrrrr}
\hline \hline Abell & $N_{\text {cells }}$ & $\theta$ & Abell & $N_{\text {cells }}$ & $\theta$ & Abell & $N_{\text {cells }}$ & $\theta$ \\
\hline & & & & & & & & \\
1408 & 6 & 89.7 & 2026 & 6 & 46.3 & 2614 & 9 & 84.4 \\
1409 & 9 & 36.2 & 2030 & 6 & 179.5 & 2630 & 7 & 167.2 \\
1412 & 5 & 20.7 & 2034 & 11 & 48.8 & 2654 & 10 & 24.1 \\
1419 & 14 & 33.2 & 2064 & 6 & 134.5 & 2656 & 14 & 26.3 \\
1423 & 11 & 130.4 & 2101 & 6 & 91.4 & 2665 & 15 & 122.1 \\
1433 & 8 & 5.6 & 2120 & 14 & 174.0 & 2678 & 5 & 70.5 \\
1437 & 5 & 58.2 & 2141 & 8 & 89.2 & 2698 & 9 & 19.1 \\
\hline
\end{tabular}


Table 2: Nearest-neighbour alignment signal and its standard deviation (column 2) as a function of limiting maximum cluster separation (column 1). Column 3 shows the signal to noise ratio, column 4 the number of separations considered and column 5 the probability, estimated from a KolmogorovSmirnov test, that the distribution of $\phi_{n n}$ 's is drawn from a uniform one.

\begin{tabular}{ccccc}
\hline \hline$D_{\text {lim }}\left(h^{-1} \mathrm{Mpc}\right)$ & $\delta \pm \sigma$ & $\delta / \sigma$ & No of pairs & $P_{K S}$ \\
\hline & & & & \\
10 & $-9.4 \pm 3.3$ & 2.9 & 61 & 0.04 \\
15 & $-6.3 \pm 2.5$ & 2.5 & 106 & 0.03 \\
30 & $-3.9 \pm 1.9$ & 2.0 & 192 & 0.22 \\
50 & $-4.3 \pm 1.6$ & 2.7 & 254 & 0.06 \\
60 & $-4.4 \pm 1.6$ & 2.8 & 260 & 0.05 \\
\hline
\end{tabular}

Table 3: All-neighbour alignment signal.

\begin{tabular}{ccccc}
\hline \hline$D_{\text {lim }}\left(h^{-1} \mathrm{Mpc}\right)$ & $\delta \pm \sigma$ & $\delta / \sigma$ & No of pairs & $P_{K S}$ \\
\hline & & & & \\
$0-10$ & $-10.9 \pm 2.9$ & 3.8 & 80 & $2 \times 10^{-3}$ \\
$10-30$ & $-2.45 \pm 1.13$ & 2.2 & 528 & 0.06 \\
$30-50$ & $-2 \pm 0.85$ & 2.3 & 904 & 0.015 \\
$50-150$ & $-1.5 \pm 0.41$ & 3.6 & 3944 & 0.01 \\
$0-150$ & $-1.8 \pm 0.35$ & 5.1 & 5456 & $10^{-4}$ \\
\hline
\end{tabular}




\section{References}

Argyres, P.C., Groth, E.J. \& Peebles, P.J.E. 1986, AJ, 91, 471

Barnes, J. \& Efstathiou, G. 1987, ApJ, 270, 20

Binggeli, B. 1982, A\&A, 107, 338

Bond, J.R. 1987, in Nearly Normal Galaxies, ed. S.Faber (NY:Springer), 388

Borgani, S., Jing, Y.P. \& Plionis, M. 1992, ApJ, 395, 339

Borgani, S., Plionis, M. \& Valdarnini, R. 1993, ApJ, 404, 21

Binney, J. \& Silk, J. 1979, MNRAS, 188, 273

Blumenthal, G.R., Faber, S.M., Primack, J.R. \& Rees, M.J. 1984, Nature, 311, 517

Carter, D. \& Metcalfe, J. 1980, MNRAS, 191, 325

Davis, M., Efstathiou, G., Frenk, C.S.\& White, S.D.M. 1985, ApJ, 292, 371

Doroshkevich, A,G., Shandarin, S. \& Saar E. 1978, MNRAS, 184, 64

Dekel, A., West, M.J., \& Aarseth, S.J. 1984, ApJ 279, 1

Dekel, A., \& West, M.J. 1985, ApJ 288, 411

Efstathiou, G., Frenk, C.S., White, S.D.M., Davis, M. 1988, MNRAS, 235, 715

Frenk, C.S., White, S.D.M., Efstathiou, G. \& Davis, M. 1985, Nature, 317, 595

Frenk, C.S., White, S.D.M., Davis, M. \& Efstathiou, G. 1988, ApJ, 327, 507

Flin, P. 1987, MNRAS, 228, 941

Fong, R., Stevenson, P.R.F. \& Shanks, T. 1990, MNRAS, 242, 146

Groth, E.J. \& Peebles, J.E., 1977, ApJ, 217, 385

van Kampen, E. \& Rhee, G.F.R. 1990, A\&A, 237, 283

Lambas, D.G., Groth, E.J. \& Peebles, P.J.E. 1988, AJ, 95, 996

Lambas, D.G., Nicotra, M., Muriel, H. \& Ruiz, L. 1990, AJ, 100, 1006

Mattig, W. 1958, Astr. Nach. 284, 109

McMillan, S.L.W., Kowalski, M.P. \& Ulmer, M.P., 1989, ApJS, 70, 723

Muriel, H., Nicotra, M., Lambas, D.G. 1991, AJ, 101, 1997

Peebles, P.J.E., 1982, ApJ, 263, L1

Plionis, M. 1988, MNRAS, 234, 401

Plionis, M., Barrow, J.D. \& Frenk, C.S.F. 1991, MNRAS, 249, 622

Plionis, M. \& Borgani, S. 1992, MNRAS, 254, 306

Plionis, M., Valdarnini, R. \& Jing, Y.P. 1992, ApJ, 398, 12

Rhee, G.F.R. \& Katgert, P. 1987, A\&A, 183, 217

Rhee, G.F.R., van Haarlem, M. \& Katgert, P. 1992, AJ, 103, 1721

Rhee, G.F.R. \& Roos, N. 1990, MNRAS, 243, 629

Rood, H.J. 1988, ARAA 26, 245

Salvador-Sole, E. \& Solanes, J.M. 1993, ApJ, 417, 427

Seldner, M., Siebers, B., Groth, E.J. \& Peebles, J.E. 1977, AJ, 84, 249

Shane, C.D. \& Wirtanen, C.A., 1967 Publs Lick Obs., XXII, Part 1 
Shectman, S., 1985, ApJS, 57, 77

Struble, M.F. \& Peebles, P.J.E. 1985, AJ, 90, 582

Struble, M.F. 1990, AJ, 99, 743

Trevese, D., Cirimele, G. \& Flin, P. 1992, AJ, 104, 935

West, M.J., Dekel, A. \& Oemler, A.Jr. 1989, ApJ 336, 46

West, M.J. 1989a, ApJ 344, 535

West, M.J. 1989b, ApJ 347, 610

West, M.J., Villumsen, J.V. \& Dekel, A. 1991, ApJ, 369, 287

Zeldovich, Ya. B., 1970, A\&A, 5, 84 


\section{Figure Captions}

Figure 1. Cluster position angles, $\theta$, as a function of the number of $10^{\prime} \times 10^{\prime}$ cells, defining the cluster sampling area, used in their estimation. The position angles estimated only at one overdensity level, are plotted.

Figure 2. As in Figure 1 but for clusters with more than one position angle determination: (a.) The average position angle, $\langle\theta\rangle$ as a function of minimum number of $10^{\prime} \times 10^{\prime}$ cells used in its estimation. (b.) $\langle\theta\rangle$ as a function of the maximum number of cells used.

Figure 3. The frequency distribution of $\phi_{n n}$ : (a.) Solid and broken lines corresponds to nearestneighbour separations of $D \leq 15$ and $\leq 10 h^{-1} \mathrm{Mpc}$, respectively. (b.) $\phi_{n n}$ distribution for all nearest-neighbour separations.

Figure 4. The frequency distribution of all-neighbour relative angles, $\phi_{i j}$, at the four indicated separation ranges. 